\title{
Research Paper: Effect of Custom-Made Insole Fabricated With Medial Heel Skive Technique on Plantar Pressure in Individuals With Patellofemoral Pain Syndrome
}

\section{Gholam Reza Aminian' ${ }^{1}$ *Fatemeh Bahramian ${ }^{1}$, Masoumeh Bagherzadeh ${ }^{1}$, Shima Fardipour ${ }^{1}$, Zahra Safaeipour ${ }^{1}$, Ameneh Abedian Avval}

1. Department of Orthotics \& Prosthetics, University of Social Welfare and Rehabilitation Sciences, Tehran, Iran.

2. Department of Orthotics \& Prosthetics, School of Rehabilitation Sciences, Isfahan University of Medical Sciences, Isfahan, Iran.

Citation: Aminian GhR, Bahramian F, Bagherzadeh M, Fardipour Sh, Safaeipour Z, Abedian Avval A. [Effect of Custom-Made Insole Fabricated With Medial Heel Skive Technique on Plantar Pressure in Individuals With Patellofemoral Pain Syndrome (Persian)]. Archives of Rehabilitation. 2016; 17(3): 252-259. http://dx.doi.org/10.21859/jrehab-1703252

http://dx.doi.org/10.21859/jrehab-1703252

Received: 01 Dec. 2015 Accepted: 20 Apr. 2016

Keywords: Patellofemoral pain syndrome, Insole, Foot orthoses, Plantar foot pressure, Pedar system

\section{A B STRACT}

Objective Knee problems, particularly patellofemoral pain syndrome (PFPS), are one of the common complaints of adolescents and young adults. PFPS is also known as "runner's knee" or "jumper's knee" due to its high prevalence among sportspersons. Its symptoms can be aggravated by ascending and descending stairs, squatting, deep squatting, kneeling, prolonged sitting, standing up from sitting, and running. Hence, it affects many aspects of daily life, including the ability to perform pain-free exercise or work-related activities. Nonsurgical treatments such as physiotherapy, insoles, and knee orthoses are commonly used to treat people with PFPS. Insoles with medial heel skive is a new method of insole modification that can control pronation and involves the selective removal of small amounts of medial plantar heel of the positive cast of the foot.

The aim of this study is to evaluate the effects of custom-made insole with medial heel skive technique on plantar pressure in PFPS subjects.

Materials \& Methods In this controlled before-after study, 14 subjects with PFPS were recruited. Plantar pressure and contact area in eight areas of the foot with and without insole with medial heel skive technique were measured using the Pedar system. A paired t-test was used for statistical analysis.

Results The results of this study showed that the use of insole with medial heel skive technique resulted in decreased plantar pressure in the medial hind foot $(\mathrm{P}=0.001)$ compared to without insole. Also, the average plantar pressure $(\mathrm{P}=0.003)$ and contact area $(\mathrm{P}=0.002)$ in the medial mid-foot with the insole with medial heel skive significantly increased in comparison to without insole. However, no significant change was observed in other regions.

Conclusion The above-mentioned insoles managed to control pronation and alter plantar pressure distribution. However, since the insoles simply transferred the pressure from one area to another area and failed to distribute the pressure equitably over the examined regions, they cannot be considered to have improved plantar pressure distribution in patients with PFPS.

\section{* Corresponding Author:}

Fatemeh Bahramian, MSc.

Address: Department of Orthotics \& Prosthetics, University of Social Welfare and Rehabilitation Sciences, Koodakyar Ave., Daneshjoo Blvd., Evin, Tehran, Iran. Tel: +98 (913) 7854164

E-Mail: fbahramian69@gmail.com 


\title{
بررسى تأثير كفى سفارشى ساختهشده بلشيوه "برداشت از بخش داخد داخلى ياشنه" بر توزيع فشار

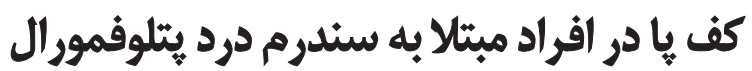

\author{
غلامرضا امينيان'، "فاطمه بهراميان'، معصومه باقرزاده'، شيما فردىيور'، زهرا صفايعيور'، آمنه عابدياناول' \\ 1- إكروها ارتويدى فنى، دانشكاه علوم بهزيستى و توانبخشى تهران، تهران، ايران.

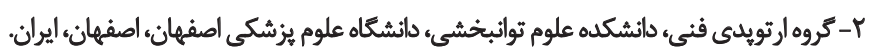

\begin{abstract}
حكيد

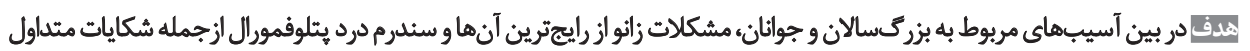

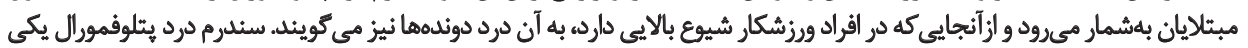

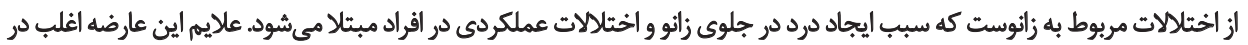

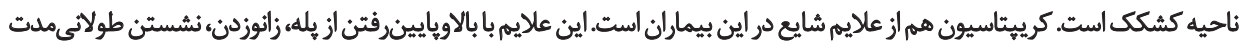

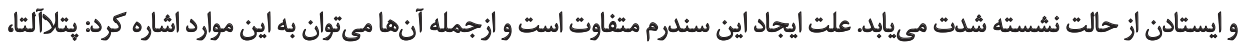

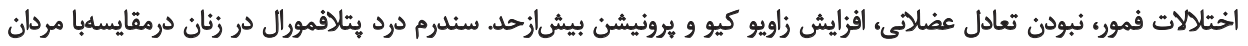

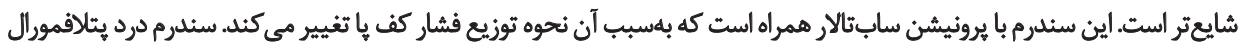

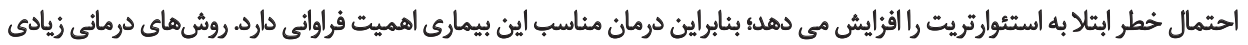

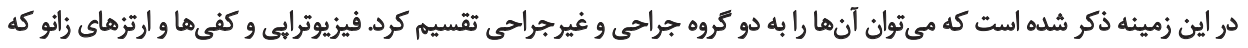

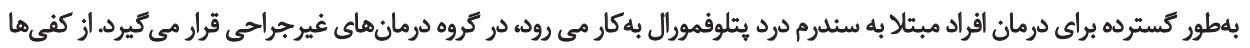

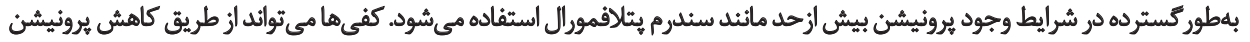

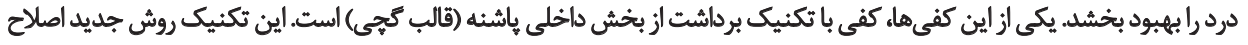

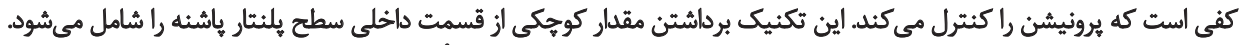

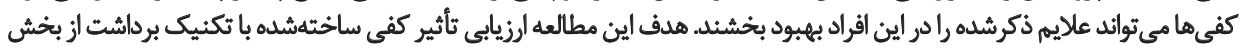

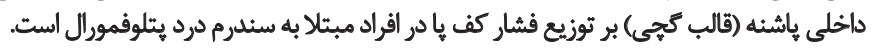

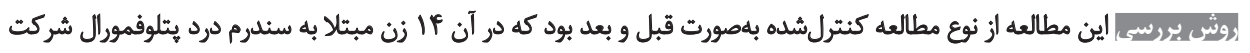

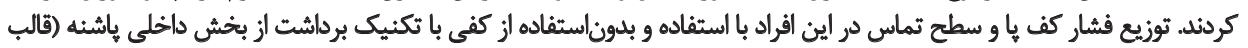

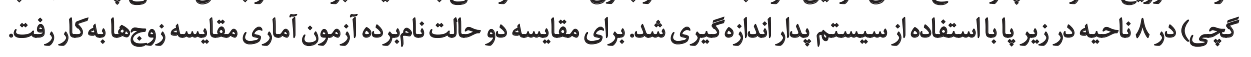

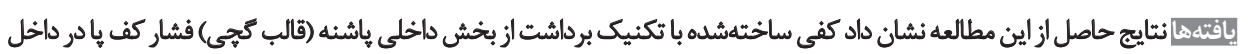

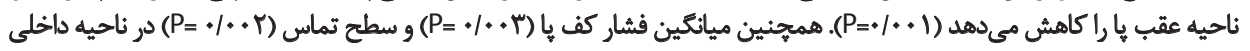

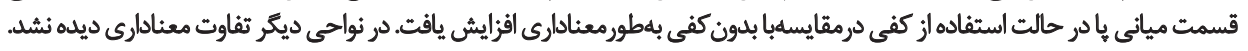

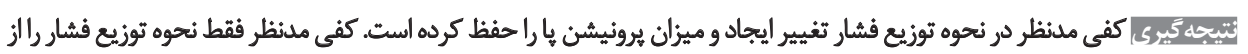

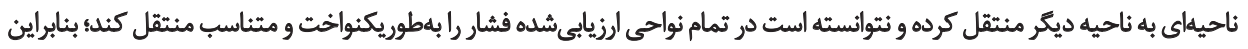

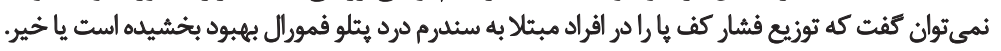

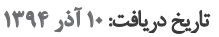

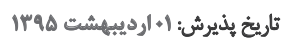

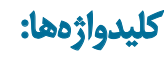

سندرم درث

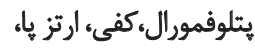

فشار كف ها، سيستم بدار

دردناك يتلوفمورال اغلب بهجاى زانوى دردناك دوندهها' نيز استفاده

مقدمه

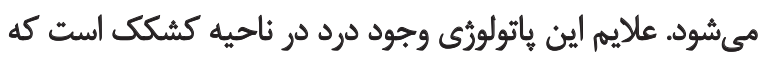

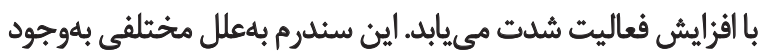

يكى از شايعترين اختلالات اسكلتىعضلانى ناحيه زانو در افراد

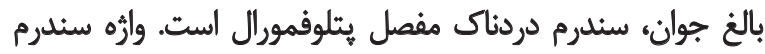

1. Runner's knee

$$
\begin{aligned}
& \text { * نويسنده مسئول: } \\
& \text { قاطمه بهراميان }
\end{aligned}
$$

نشانى: تهران، اوين، بلواردانشجو، بنيست كودكيار، دانشكاه علوم بهزيستى و توانبخشى، كروه ارتويدى فنى.

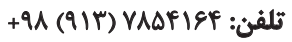
رايانامه: fbahramian69@gmail.com 
كاهش دهد و از اين طريق الكوى توزيع فشار را در افراد دجار

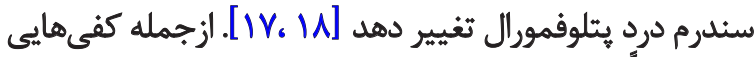

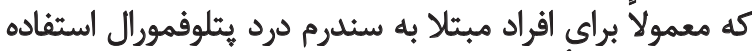

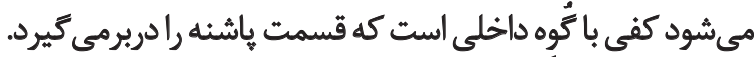

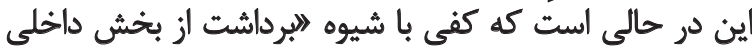

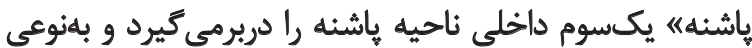

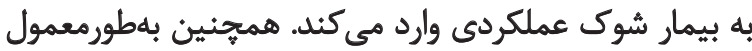

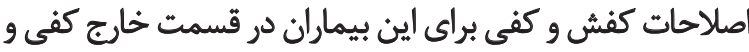

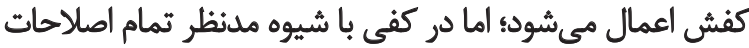

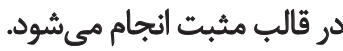

اين شيوه حجم كلى كفى را بهمنظور اندازه مطلوب در كفش

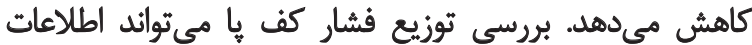

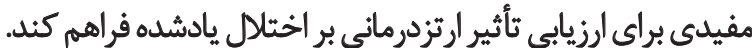

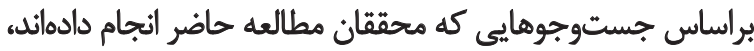

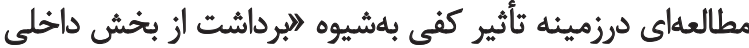

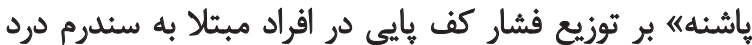

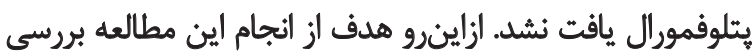

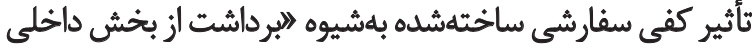

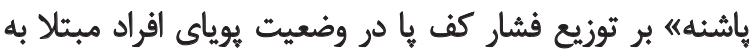

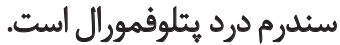

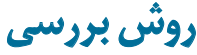

در اين مطالعه كه بلصورت مطالعه كنترلشده قبل و بعد انجام

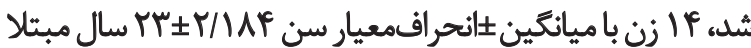

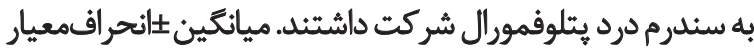
قد و وزن شركتكنيدكان بهترتيب

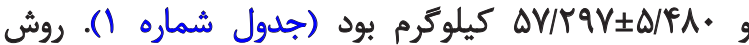

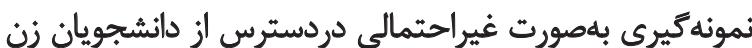

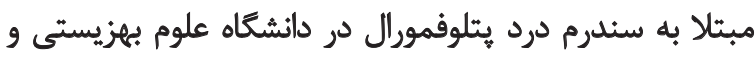

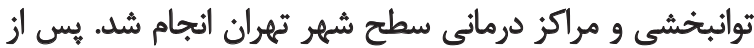

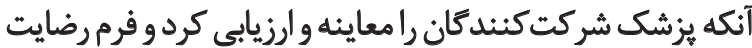

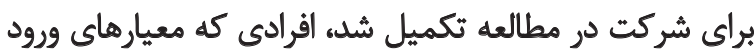

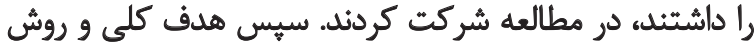
مطالعه براى افراد شرح داده شد.

معيارهاى انتخاب افراد در اين مطالعه عبارث بود ازي: ابتلا به

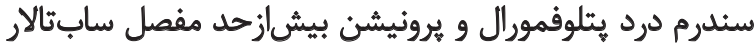

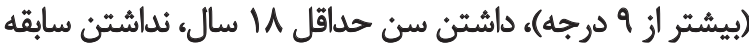

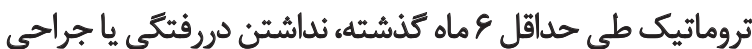

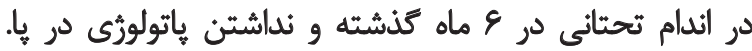

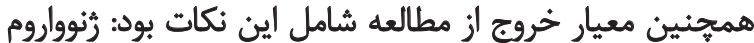

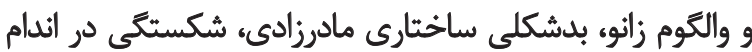

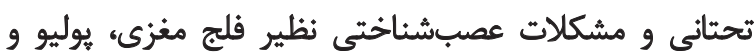
اسيينابيفيدا و رضايتنداشتن بيمار براى شركت در مطالعه.
مي آيد. اين علل عبارت است از: تروما، اختلالات مفصل زانو مانيد

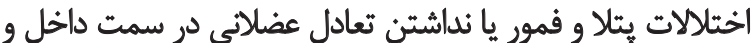

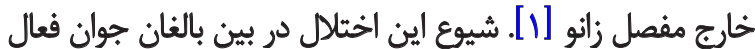

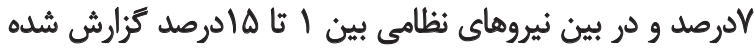

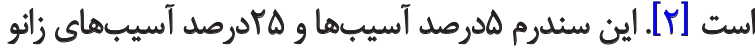

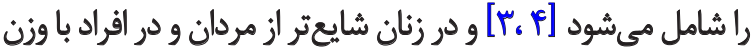

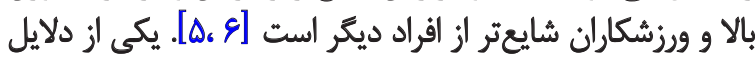

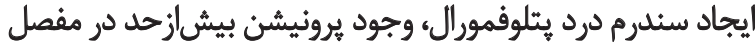

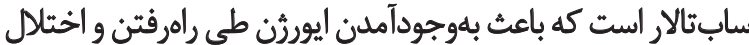

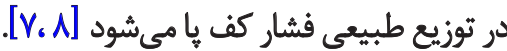

در راهفتن عادى و در ابتداى مرحله ايستايى قبل از برخورد

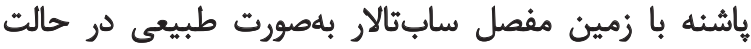

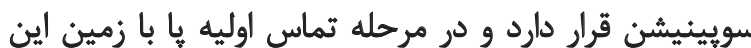

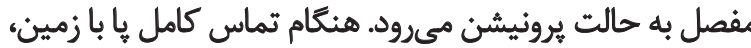

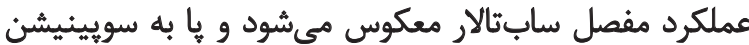

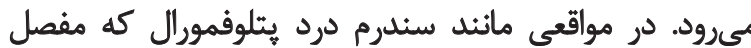

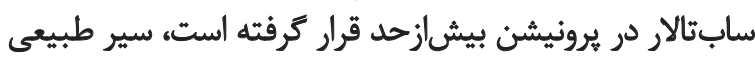

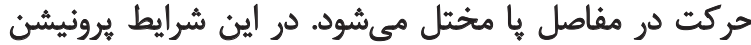

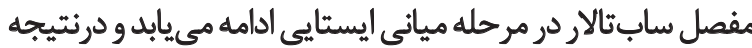

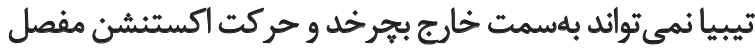

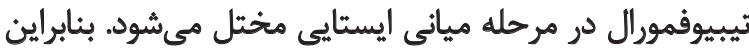

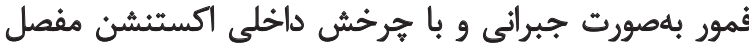

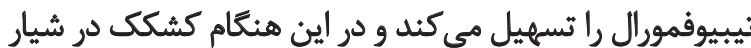

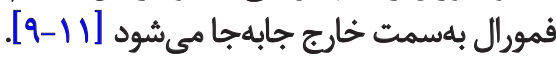

مطالعات نشان مىدهد اين عارضه بلهعلت اختلال در راستاى

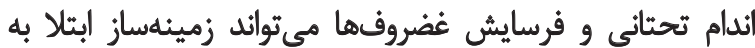

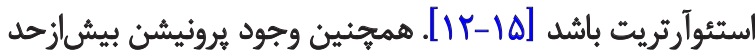

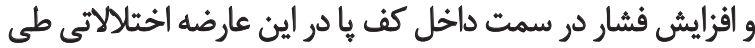

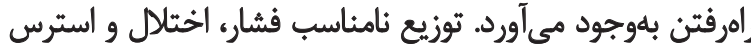

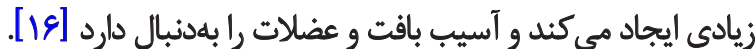

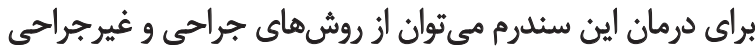

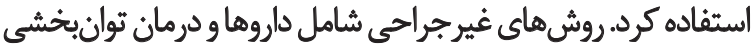

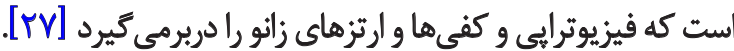

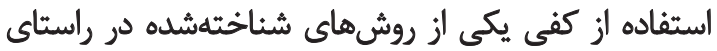

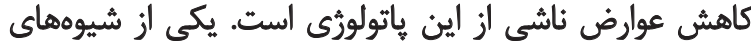

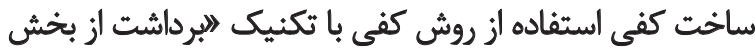

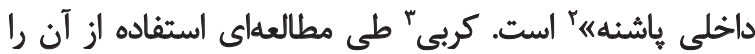

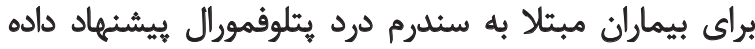

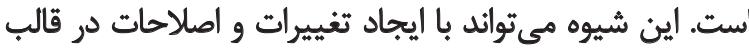

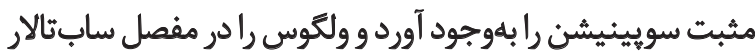

2. Medial Heel Skive

3. Kirby 


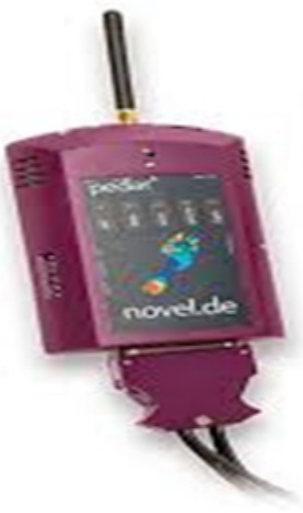

توانبخنتى

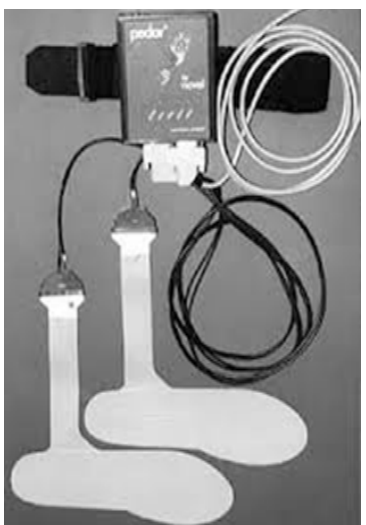

موردمطالعه كفش همه شركت كنندكان استاندارد و از يك مدل

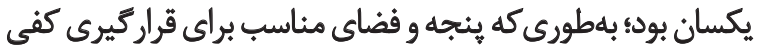

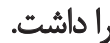

درراستاى تجزيهوتحليل اطلاعات سطح كف با إز طريق بريق

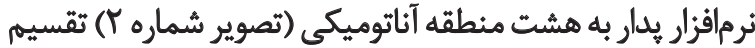

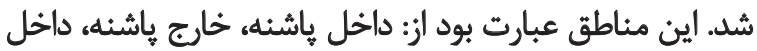

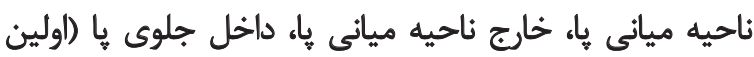

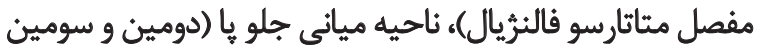

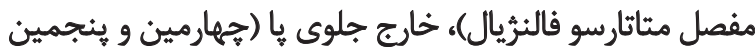

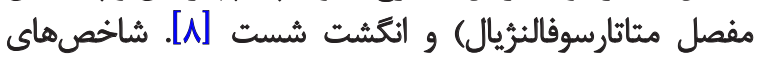

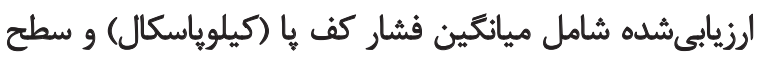

$$
\text { تماس (سايتتىمتر) بود. }
$$

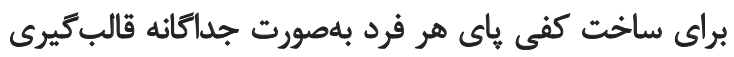

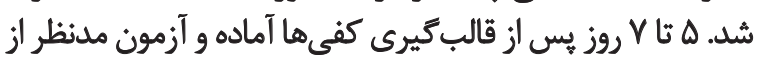

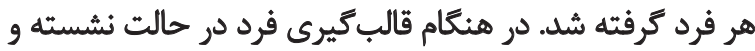

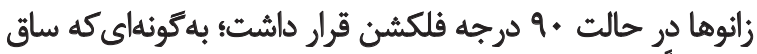

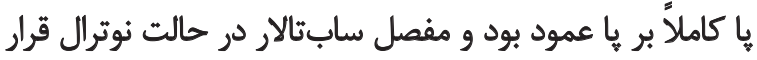

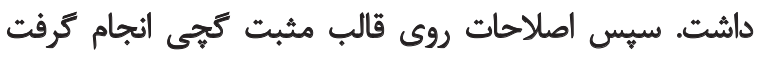
(تصوير شماره ؟). در اين شيوه يكسوم داخلى سطح كف باتيى

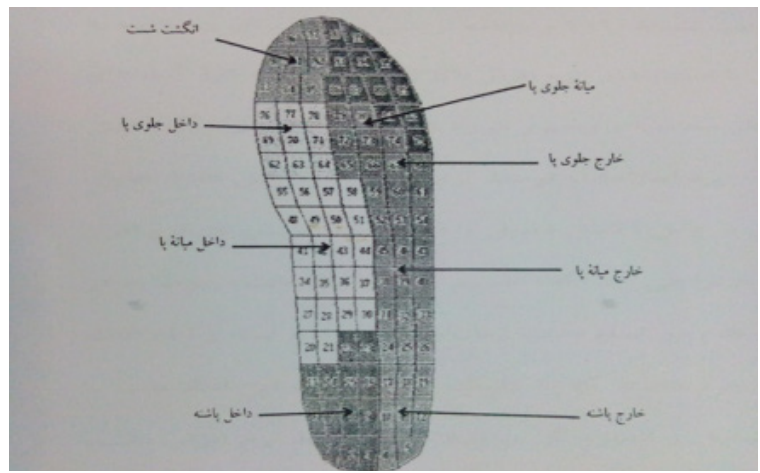

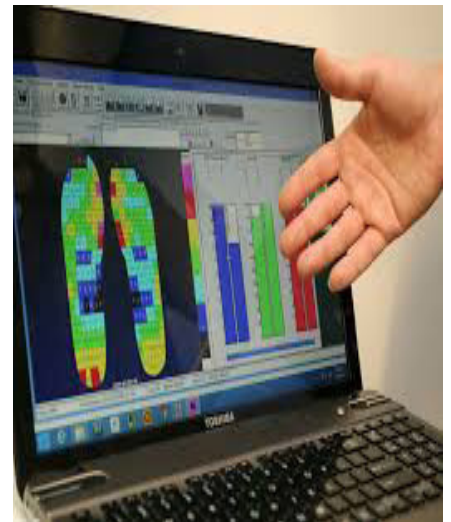

تصوير ا. سيستم بدار.

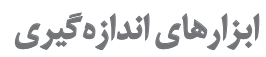

بلمنظور تعيين ميزان يرونيشن سابتالار و اندازهيرى زاويه

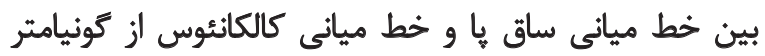

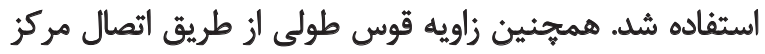

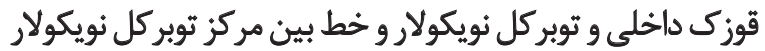

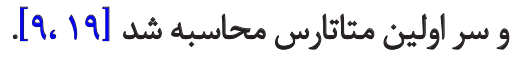

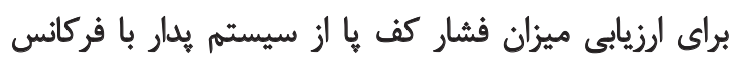

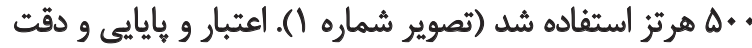

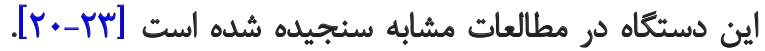

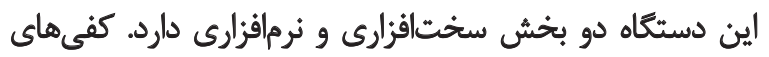

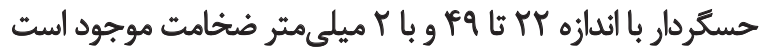

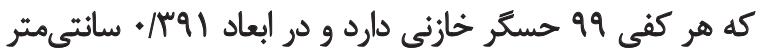

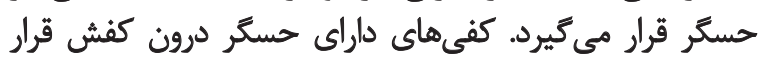

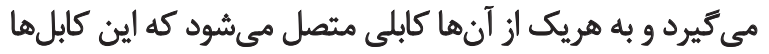

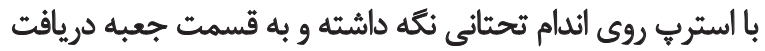

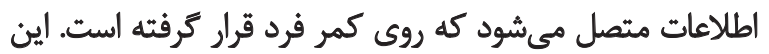

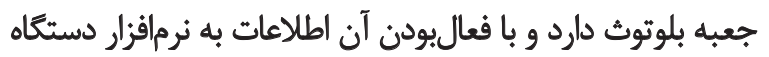

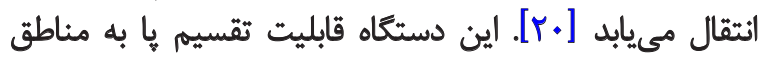

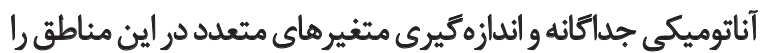

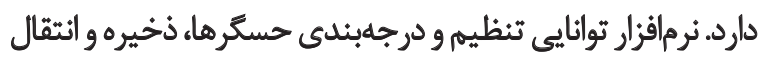

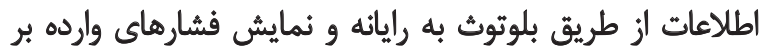

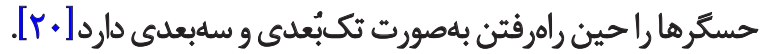

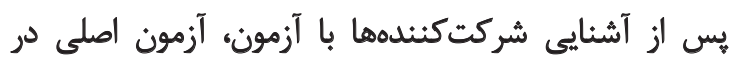

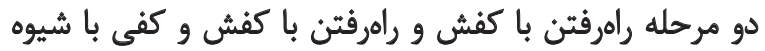

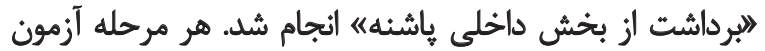

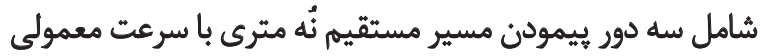

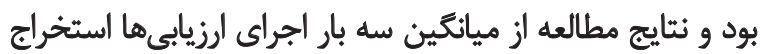

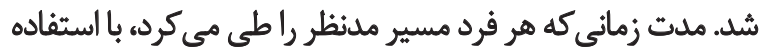

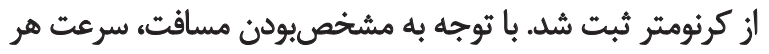

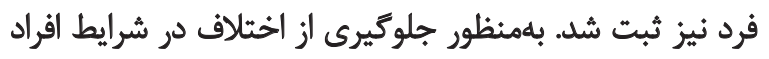


كفى با ضخامت بالاى هيهارونيم ميلىمتر و براي افراد با وزن زير

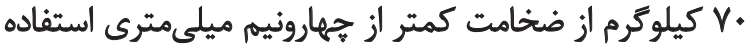

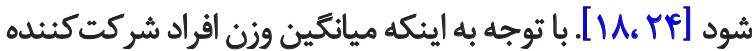

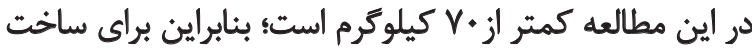

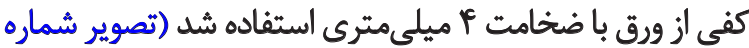

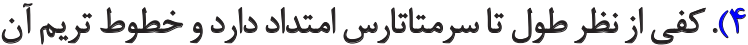

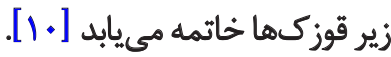

تحليل آمارى دادهها

SPSS تجزيهوتحليل آمارى دادهها با كمك نسخه • ب نرمافزار

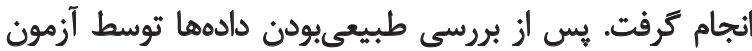

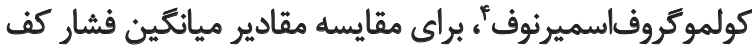

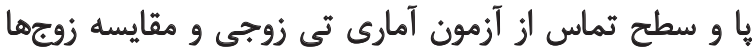

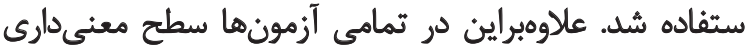

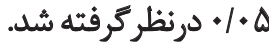

\section{يأثتلها}

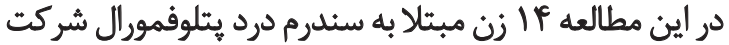

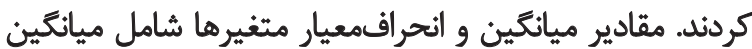

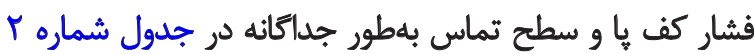

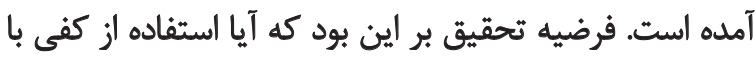

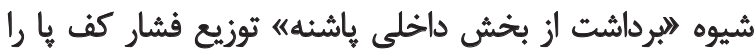

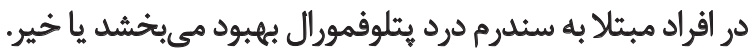

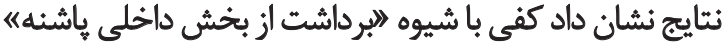

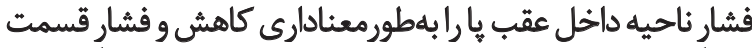

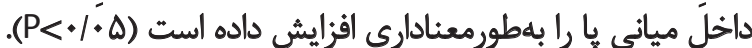

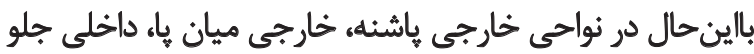

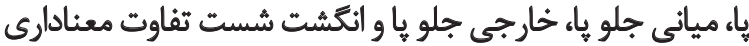

همجنين كفى با شيوه البرداشت از بخش داخلى باشئه) در

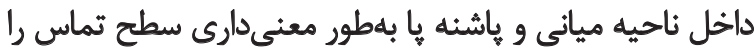

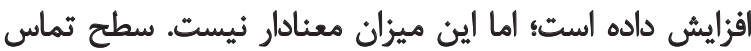

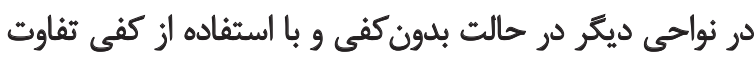

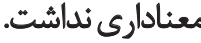

4. Kolmogrov-Smirnov

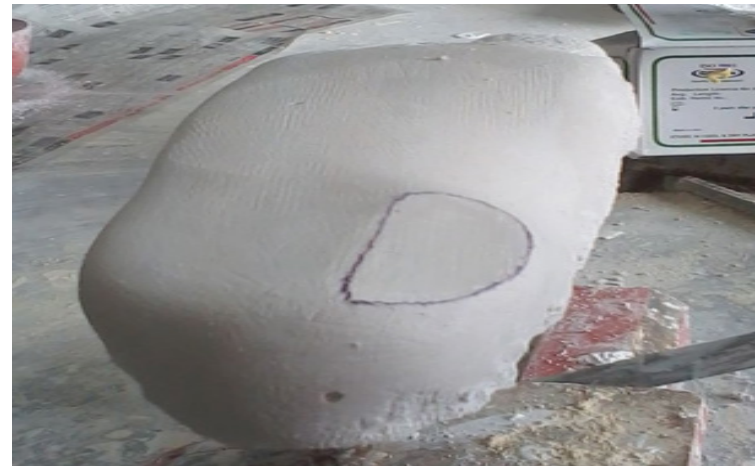

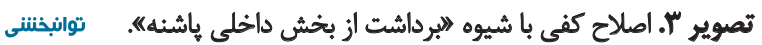

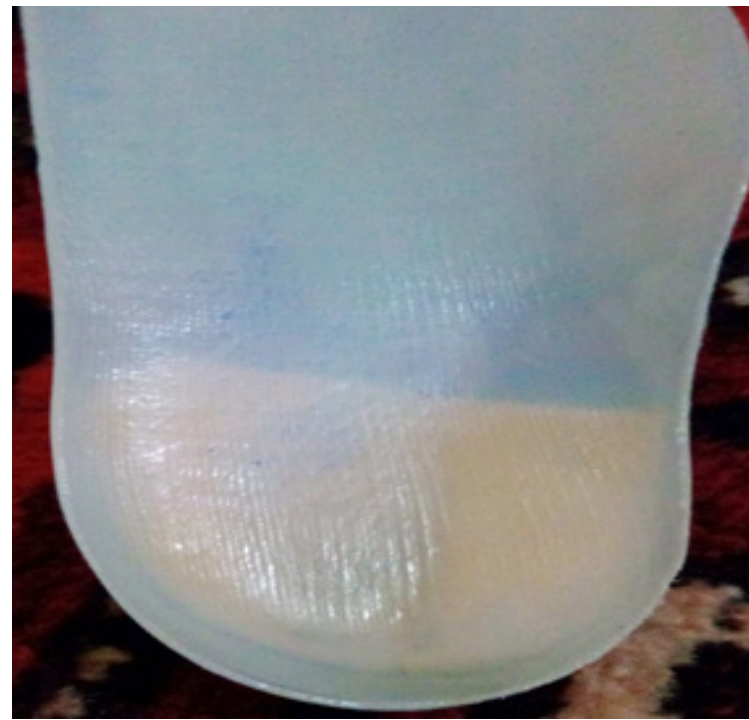

توانبخننى

تصوير f. اصلاح كفى با شيوه "ليرداشت از بخش داخلي ياشنهي.

در پاشنه توسط كوليس و خطكش طي مرحله قالب بيرى و

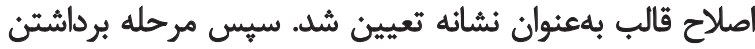

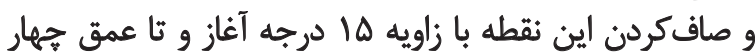

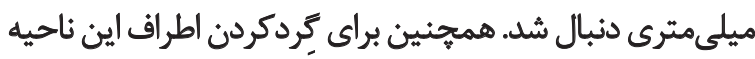

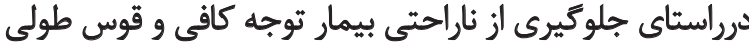

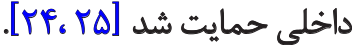
يشنهاد شده است براى افراد با وزن كمتر از • V كيلوكرم از

جدول ا. ميانَّين و انحرافُ استاندارد نمودار آزمودنىها در اطلاعات شخصي.

\begin{tabular}{|c|c|c|}
\hline اتحرافمعيار & مياتكين & مثغيرها \\
\hline T/MF & M & سن (سال) \\
\hline$\Delta / \uparrow \Lambda$ & $\Delta V / T q Y$ & وزن (كيلوكرم) \\
\hline$\Delta / F \lambda$ & $18 M / N T$ & قد (سانتىمثر) \\
\hline
\end{tabular}




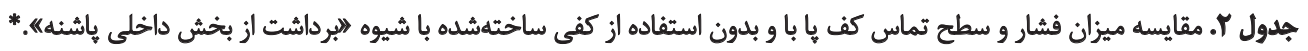

\begin{tabular}{|c|c|c|c|c|c|}
\hline سطح معثادار & اختلاف ميانكين & با كفى & بدون كفى & & 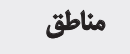 \\
\hline $.1 \cdot 01^{\circ}$ & $\mathbb{W} \cdot q$ & $\| r \cdot / 19 T \pm r \mid / N \Delta$ & IFNTAYETE/OT & ميانكين (كيلوانحرالفمعيار فشار) & \\
\hline.$/ 4$ & $\cdot / \pi r$ & $1 \Delta / 7 \Delta \pm \cdot / \cdot q T$ & $\mid Q / \cdot r \pm 1 / E T$ & ميانكين (انحرافتمعيار سطح تماس & داخل ياشنه \\
\hline $.11 \cdot 4$ & $\Delta / F T$ & $1 r \cdot / 1 q T \pm r g / \Delta f$ & $I M E / N A S \pm r V / W$ & ميانكين (كيلوانحرافلامعيار فشار & خارج ياشئه \\
\hline$\cdot / 1$ &.$/ 11$ & $19 / P f \pm T / N A$ & IV/TE士Y/N & ميانكين دانتحرافـمعيار سطح تماس & \\
\hline .0. & $1 \% / 94$ & $M r / Q \Psi \pm I Y / l a$ & $V / \cdot 1 \pm 2 / \pi$ & 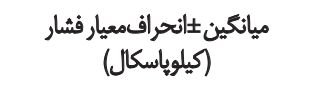 & داخل ناحيه \\
\hline$\%$ & V/TT & $17 / f+q 9 \pm \Delta / 19$ & $\Delta / r \cdot Y \pm \psi / T q$ & هيانكين \انحر|فمعيار سطع تماس & ميانى ها \\
\hline .MIA & $T / N T A$ & $\Delta f / \cdot M \pm(Q / \lambda \mid$ & $\Delta V / r \cdot q \pm Y r / M$ & 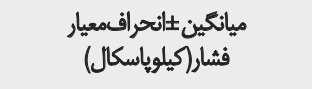 & خارج ناهيه \\
\hline 1.8 & $r /+Y$ & $r=/ Q \Delta \pm \& / V q$ & $W M \cdot \pm \Delta / M$ & مياتكين دانترافمعيار سطع تماس & ميانى ها \\
\hline.$/ 481$ & $r / F \Delta$ & $\mid r \cdot / u \pm F+/ F$ & $\| V / \Delta T \pm F \mid M$ & 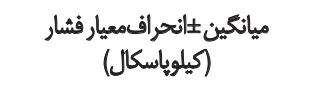 & \\
\hline.$/ M$ & V/TE & $\mid r / F q \pm r / F \Delta$ & $W / \pi \Psi \pm 1 / \%$ & ميائكين 1 (سنحرافتمعيار سطع تماس & \\
\hline . IATY & $\varphi / \Delta \& \Delta$ & $|f \cdot M| \pm r \Delta / r \Lambda$ & $\mid T E / T \Delta \pm Y \Delta / T$ & ميانكيند|نحرافلفمعيار فشارار & \\
\hline.$/ 18$ & $+18+0$ & IVIEAEVIPF & $|V| \cdot V \Delta \pm 1 / T r$ & ميانكين \$انحرافمعيار سطع تماس & ميان جلوى ها \\
\hline.$/ F N$ & ir & ITT/MTIETI/V+r & $I T V / Y+Y \pm Y_{+} / I T+$ & هيانكين فُشار (كيلوياسكال) & \\
\hline.$/ 11$ & . $/ \Delta I F$ & W/AAY $\pm V / Y q$ & $M / T H A \pm V / M \Delta$ & سطح تماس (سائتىمتر) & \\
\hline.$/ T W$ & NUT & $r W D+Y \pm Y_{+} / r_{+}$ & $r+q / \Delta Y \pm F Y /+r$ & هيانكين فُشار (كيلوياسكال) & تكشت شست \\
\hline .199 & .1 .9 & $N G+ \pm \cdot / 9 F$ & $N(D) \pm N E$ & سطع تماس (سائتىمتر) & \\
\hline
\end{tabular}

توانبخننى

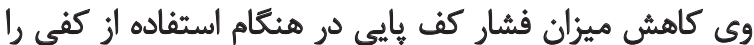

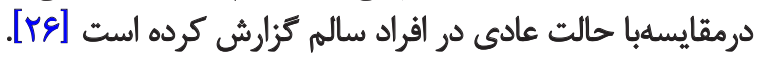

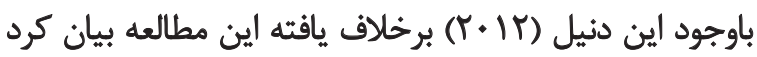

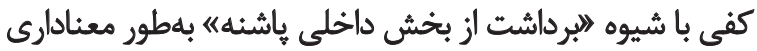

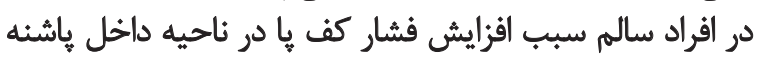
مي شود أراد سالم

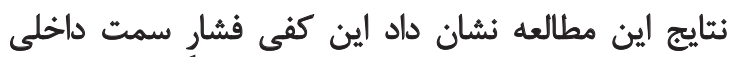

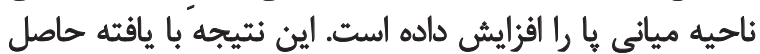

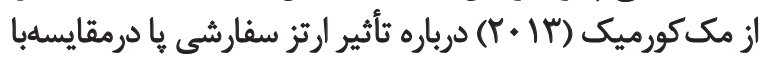

هدف از انجام اين مطالعه بررسى ثأثير كفى سفارشى

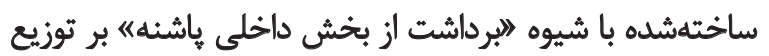

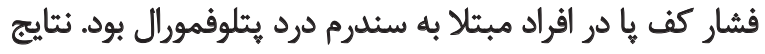

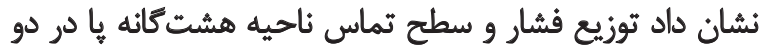

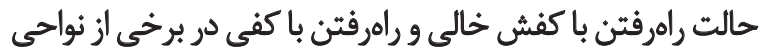

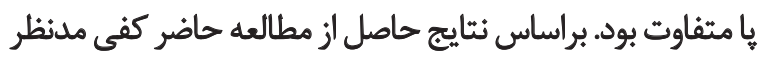

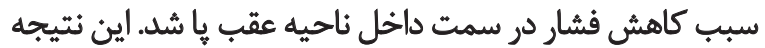

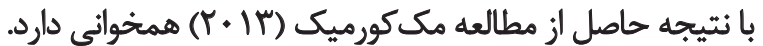


نواحى ارزيابى شُه فشار رابهطور يكنواخت و متناسب منتقل كند؛

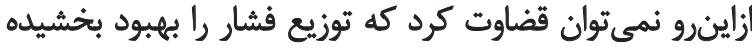

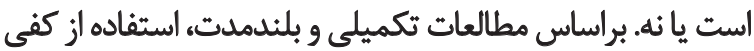

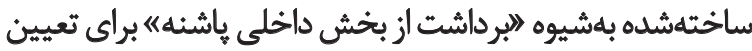

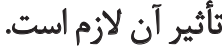

مجدوديثها

تهمهرتين محدوديتهاى مطالعه حاضر عبارت بود ازئ كمبودن

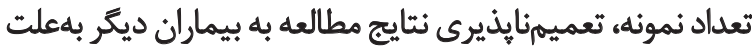

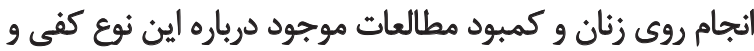

$$
\text { نياز به آزمون آن. }
$$

بيشنهادها

با توجه به بررسي تأثير آنى در اين مطالعه بيشنهادهاى زير ارائه مي شود:

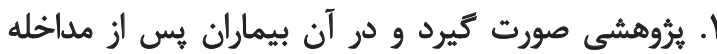
بلندمدت از نظر فشار كف بائي بررسي شوردي بيشتر درباره مؤثربودن كفى مدنظر اظظهار بنظر كردي؛ r. تحقيق حاضر روى هر دو جنس (زن و مرد) با نمونه آمارى

$$
\text { بيشترى انجام شود؛ }
$$

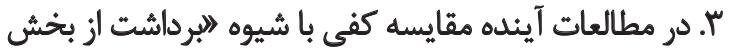

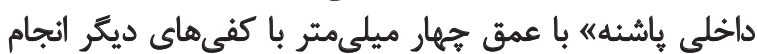

$$
\text { تشكر و قدردانى }
$$

اين مقاله بركرفته از باياننامه كارشناسى ارشد خانم فاطمه

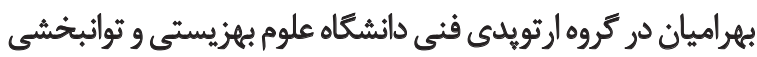

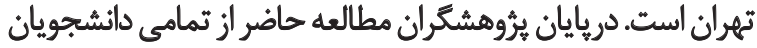

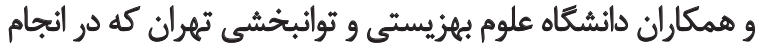
اين مطالعه شركت و همكارى داشتند، تشكر و قدردانى مى كثنئد.
كفُشهاى عادى مشابه بود. درنتيجه اين كفى از طريق انثقال

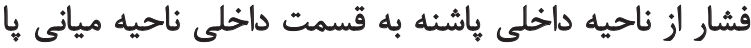

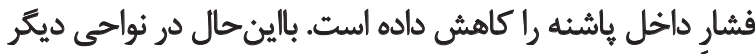

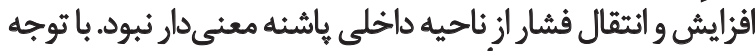

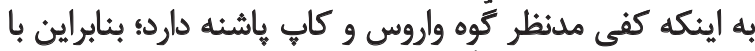

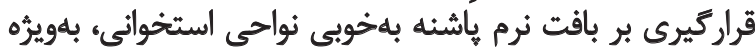

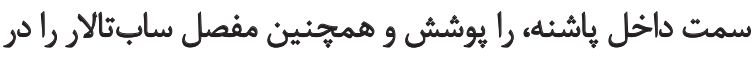

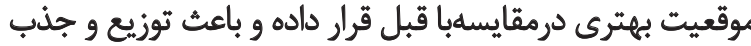
بهتر فشار در اين ناحيه شده است. با وجود اينكه يرونيشن بيشازحد همراه با سندرم درد

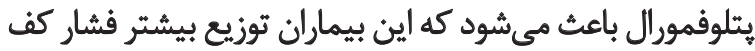

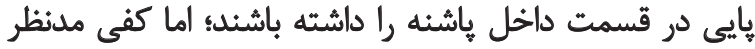

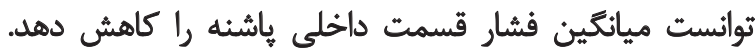

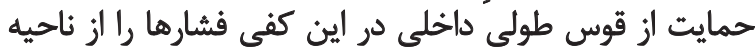

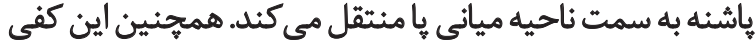

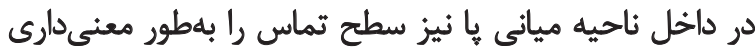

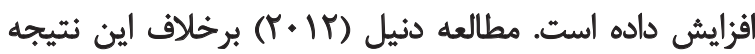

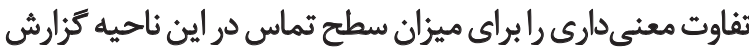

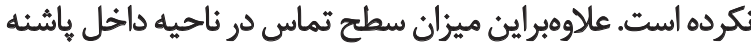

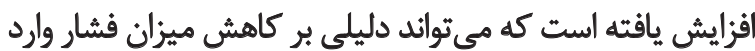

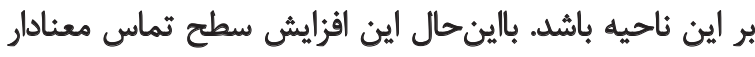

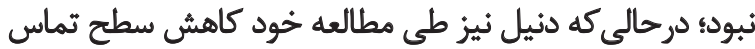

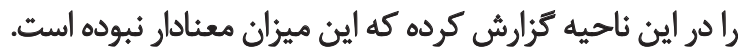

اترجه در مطالعات ديكر بهبود علايم افراد مبتلا به سندرم

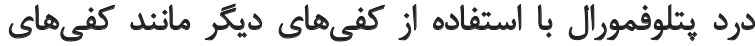

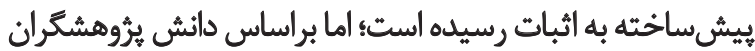

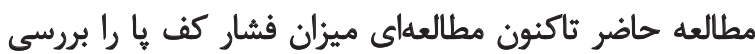

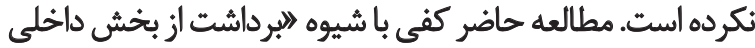

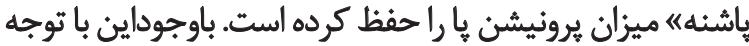

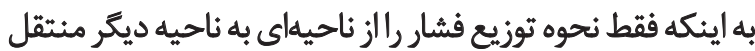

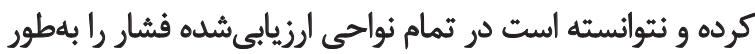

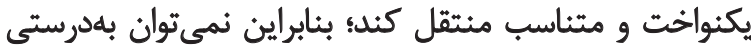

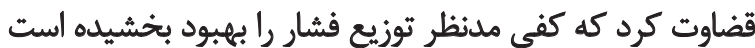

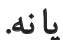

\section{تتيجليرى}

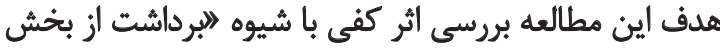

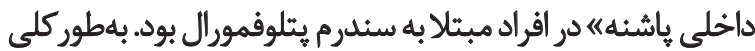

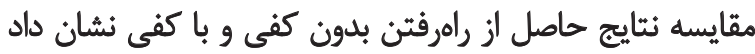

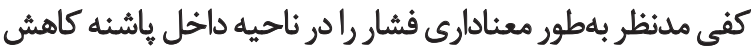

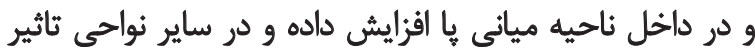

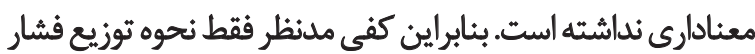

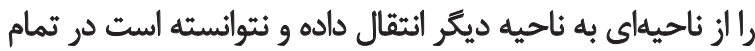




\section{References}

[1] Dixit S, Difiori JP, Burton M, Mines B. Management of patellofemoral pain syndrome. American Family Physician. 2007; 75(2):194-202.

[2] Shwayhat AF, Linenger JM, Hofherr LK, Slymen DJ, Johnson CW. Profiles of exercise history and overuse injuries among United States Navy Sea, Air, and Land (SEAL) recruits. American Journal of Sports Medicine. 1994; 22(6):835-40. doi: 10.1177/036354659402200616

[3] Baker MM, Juhn MS. Patellofemoral pain syndrome in the female athlete. Clinics in Sports Medicine. 2000; 19(2):315-29.

[4] Nejati P, Forogh B, Moeineddin R, Baradaran H, Nejati M. Patellofemoral pain syndrome in Iranian female athletes. Acta Medica Iranica. 2010; 49(3):169-72.

[5] Dugan SA. Sports-related knee injuries in female athletes: what gives? American Journal of Physical Medicine \& Rehabilitation. 2005; 84(2):122-30. doi: 10.1097/01.phm.0000154183.40640.93

[6] Peterson L, Renström P, Grana WA. Sports injuries: their prevention and treatment: Chicago: Year Book Medical Publishers; 1986.

[7] Klingman RE, Liaos SM, Hardin KM. The effect of subtalar joint posting on patellar glide position in subjects with excessive rearfoot pronation. Journal of Orthopaedic \& Sports Physical Therapy. 1997; 25(3):185-91. doi: 10.2519/jospt.1997.25.3.185

[8] Bonanno DR, Zhang CY, Farrugia RC, Bull MG, Raspovic AM, Bird AR, et al. The effect of different depths of medial heel skive on plantar pressures. Journal of Foot and Ankle Research. 2012; 5(1):9. doi: 10.1186/1757-1146-5-s1-o9

[9] Tiberio D. The effect of excessive subtalar joint pronation on patellofemoral mechanics: a theoretical model. Journal of Orthopaedic \& Sports Physical Therapy. 1987; 9(4):160-5. doi: 10.2519/ jospt.1987.9.4.160

[10] Aliberti S, Costa SX, Campos Passaro A, Arnone AC, Hirata R, Sacco IC. Influence of patellofemoral pain syndrome on plantar pressure in the foot rollover process during gait. Clinics. 2011; 66(3):367-72. doi: 10.1590/s1807-59322011000300001

[11] Barton CJ, Bonanno D, Levinger P, Menz HB. Foot and ankle characteristics in patellofemoral pain syndrome: a case control and reliability study. Journal of Orthopaedic \& Sports Physical Therapy. 2010; 40(5):286-96. doi: 10.2519/jospt.2010.3227

[12] Berry PA, Teichtahl AJ, Wluka AE, Cicuttini FM. The role of biomechanical factors on patellofemoral osteoarthritis. Current Rheumatology Reviews. 2007; 3(2):123-7. doi: $10.2174 / 157339707780619368$

[13] Myer GD, Ford KR, Barber Foss KD, Goodman A, Ceasar A Rauh MJ, et al. The incidence and potential pathomechanics of patellofemoral pain in female athletes. Clinical Biomechanics. 2010; 25(7):700-7. doi: 10.1016/j.clinbiomech.2010.04.001

[14] Thomas MJ, Wood L, Selfe J, Peat G. Anterior knee pain in younger adults as a precursor to subsequent patellofemoral osteoarthritis: a systematic review. BMC musculoskeletal disorders. 2010; 11:201. doi: 10.1186/1471-2474-11-201
[15] Utting M, Davies G, Newman J. Is anterior knee pain a predisposing factor to patellofemoral osteoarthritis? The Knee. 2005; $12(5): 362-5$

[16] Safaei-Pour Z, Ebrahimi E, Saeedi H, Kamali M. Invesigation of dynamic plantar pressure distribution in healthy adults during standing and walking (Persian)]. Archives of Rehabilitation. 2009, 10(2):8-15

[17] Home P. Modification tips: making sure the shoe fits [Internet]. Pediatry Today. 2008; 15(8). Available from: http://www.podiatrytoday.com/article/ 674

[18] Kirby KA. The medial heel skive technique. Improving pronation control in foot orthoses. Journal of the American Podiatric Medical Association. 1992; 82(4):177-88. doi: 10.7547/8750731582-4-177

[19] Johnston LB, Gross MT. Effects of foot orthoses on quality of life for individuals with patellofemoral pain syndrome. Journal of Orthopaedic \& Sports Physical Therapy. 2004; 34(8):440-8. doi: 10.2519/jospt.2004.34.8.440

[20] Ramanathan AK, Kiran P, Arnold GP, Wang W, Abboud R]. Repeatability of the Pedar-X in-shoe pressure measuring system. Foot and Ankle Surgery. 2010; 16(2):70-3. doi: 10.1016/j. fas.2009.05.006

[21] Partovifar M, Safaeipour Z, Vahab Kashani R, Zabihi Yeganeh M, Bagherzadeh MS. [Immediate effect of foot insole with longitudinal medial arch support and metatarsal pad on plantar pressure distribution in females with rheumatoid arthritis (Persian)]. Archives of Rehabilitation. 2014; 15(3):72-8.

[22] Farjad Pezeshk A, Sadeghi H, Farzadi M. [Comparison of plantar pressure distribution and vertical ground reaction force between dominant and none-dominant limb in healthy subjects using Principle Component Analysis (PCA) technique. (Persian)] Archives of Rehabilitation. 2013; 14(1):91-102.

[23] Boyd LA, Bontrager EL, Mulroy SJ, Perry J. The reliability and validity of the novel Pedar system of in-shoe pressure measurement during free ambulation. Gait \& Posture. 1997; 5(2):165.

[24] Kirby KA. Evolution of foot orthoses in sports. Athletic footwear and orthoses in sports medicine. New York: Springer; 2010

[25] Kirby KA. Subtalar joint axis location and rotational equilibrium theory of foot function. Journal of the American Podiatric Medical Association. 2001; 91(9):465-87. doi: 10.7547/87507315-91$9-465$

[26] McCormick CJ, Bonanno DR, Landorf KB. The effect of customised and sham foot orthoses on plantar pressures. Journal of Foot \& Ankle Research. 2013; 6:19. doi: 10.1186/1757-1146-6-19

[27] Miri Abyaneh H, Mosallanezhad Z, Mohammadalizade H, Bakhshi E, Vahedi GH, Nourbakhsh MR. Physiotherapy with and without Superficial Dry Needling Affects Pain and Muscle Strength in Patients with Patellofemoral Pain Syndrome. 2016; 14(1):23-30 